\title{
Differential Effects of Nasal Inflammation and Odor Deprivation on Layer-Specific Degeneration of the Mouse Olfactory Bulb
}

\author{
Sanae Hasegawa-Ishii, ${ }^{1}$ Fumiaki Imamura, ${ }^{2}$ Shin Nagayama, ${ }^{3}$ Makiko Murata, ${ }^{1}$ and \\ Atsuyoshi Shimada ${ }^{1}$
}

https://doi.org/10.1523/ENEURO.0403-19.2020

${ }^{1}$ Pathology Research Team, Faculty of Health Sciences, Kyorin University, Mitaka, Tokyo 181-8612, Japan, ${ }^{2}$ Department of Pharmacology, Penn State College of Medicine, Hershey, PA 17033-0850, and ${ }^{3}$ Department of Neurobiology and Anatomy, McGovern Medical School at the University of Texas Health Science Center at Houston, Houston, TX 00730-1501

\begin{abstract}
Harmful environmental agents cause nasal inflammation, and chronic nasal inflammation induces a loss of olfactory sensory neurons (OSNs) and reversible atrophy of the olfactory bulb (OB). Here, we investigated the mechanisms underlying this inflammation-induced $\mathrm{OB}$ atrophy by histologically and biochemically comparing the OB changes in mouse models of nasal inflammation and odor deprivation. In addition, we examined whether odor stimulation is necessary for OB recovery from atrophy. One group of adult male C57BL/6 mice was administered lipopolysaccharide (LPS) unilaterally for 10 weeks to induce nasal inflammation (control animals received saline), and a second group received unilateral naris closures (NCs) for 10 weeks of odor deprivation. The OBs atrophied in both models, but odor deprivation shrank the glomerular, external plexiform, mitral, and granule cell layers (GCLs), whereas the olfactory nerve, glomerular, and external plexiform layers (EPLs) atrophied as a result of nasal inflammation. Additionally, nasal inflammation, but not odor deprivation, caused neuroinflammation in the $\mathrm{OB}$, inducing glial activation and elevated expression of interleukin-1 $\beta$ (IL-1 $\beta)$ and TNF $\alpha$. After 10 weeks of nasal inflammation, mice were housed for another 10 weeks with no additional treatment or with unilateral NC. Nasal inflammation and glial activation subsided in both groups, but glomerular and EPLs recovered only in those with no additional treatment. Our findings demonstrate that nasal inflammation and odor deprivation differentially induce layer-specific degeneration in the $\mathrm{OB}$, that loss of OSN activity rather than neuroinflammation is a major cause of inflammation-induced $\mathrm{OB}$ atrophy, and that odor stimulation is required for $\mathrm{OB}$ recovery from atrophy.
\end{abstract}

Key words: atrophy; nasal inflammation; neuro-inflammation; odor deprivation; olfactory bulb; olfactory system

\section{Significance Statement}

Chronic nasal inflammation causes a loss of olfactory sensory neurons (OSNs) and atrophy of the olfactory bulb $(\mathrm{OB})$, which recovers when inflammation subsides. To reveal the mechanisms underlying inflammation-induced $\mathrm{OB}$ atrophy, we compared the histologic and biochemical changes in OBs in mouse models of nasal inflammation and odor deprivation. In addition, we examined whether odor stimulation was required for the recovery of the OB from atrophy. Our findings revealed that nasal inflammation and odor deprivation differentially induce layer-specific degeneration in the $\mathrm{OB}$, that loss of OSN activity rather than neuroinflammation is a major cause of inflammation-induced $\mathrm{OB}$ atrophy, and that odor stimulation is required for the OB to recover from atrophy.

Received October 3, 2019; accepted March 16, 2020; First published March 20, 2020.

The authors declare no competing financial interests.
Author contributions: S.H.-I. and F.I. designed research; S.H.-I. and M.M. performed research; S.H.-I. and M.M. contributed unpublished reagents/analytic tools; S.H.-I. and F.I. analyzed data; S.H.-I., F.I., S.N., and A.S. wrote the paper. 


\section{Introduction}

Rhinitis, sinusitis, and rhinosinusitis are common diseases often caused by exposure to environmental allergens, such as bacteria, viruses, molds, and pollens (Ahmad and Zacharek, 2008; Imamura and HasegawaIshii, 2016). A major symptom of these diseases is nasal inflammation that causes nasal congestion and/or a runny nose. However, nasal inflammation is also associated with many neurologic disorders. For example, anxiety and depression are more prevalent in patients with rhinitis than in healthy subjects (Kim et al., 2016; Schlosser et al., 2016; Bedolla-Barajas et al., 2017). Moreover, olfactory dysfunction, as well as a runny nose, is a common and early sign of neurodegenerative diseases such as Parkinson's and Alzheimer's diseases (Chou et al., 2011; Doty, 2012; Daulatzai, 2015), and patients with depression exhibit reduced olfactory performance (Yuan and Slotnick, 2014; Siopi et al., 2016; Rochet et al., 2018). However, the mechanisms connecting nasal inflammation with neurologic disorders are still an enigma. As agents and factors in the nasal passage can bypass the bloodbrain barrier (Doty, 2008; Dando et al., 2014), it is important to understand how nasal inflammation affects brain structure and functions.

Chronic rhinosinusitis, as well as Parkinson's disease and depression, has been linked with reduced olfactory bulb (OB) volume (Rombaux et al., 2008; Negoias et al., 2010; Li et al., 2016; Rottstädt et al., 2018). A previous study showed that chronic nasal inflammation induced by intranasal lipopolysaccharide (LPS) administration causes glial activation and a loss of synapses in the OB within three weeks (Hasegawa-Ishii et al., 2017). Prolonged inflammation (10 weeks or more) results in atrophy of the $\mathrm{OB}$, which recovers once the inflammation resolves (Hasegawa-Ishii et al., 2019). The atrophy is attributed to the shrinkage of superficial layers of the $O B$, namely, the olfactory nerve layer (ONL), glomerular layer (GL), and external plexiform layer (EPL), especially the superficial EPL (sEPL; Hasegawa-Ishii et al., 2019). The shrinkage of the ONL and GL results from the loss of olfactory sensory neuron (OSN) axons and damage to the olfactory epithelium (OE) from persistent nasal inflammation. However, the reason for the loss of the EPL is largely unknown, because OSN axons do not penetrate this layer comprising the secondary dendrites of projection neurons, including mitral and tufted cells, which synapse with granule cell dendrites. Further studies are needed to determine the

This study was supported by the Grant-in-Aid for Scientific Research KAKENHI 18K07395 (to S.H.-I.), the National Institutes of Health Grant R01DC016307 (to F.I.), and a grant from Kyorin University.

Acknowledgements: We thank Risa lidaka, Chiaki Sasagawa, Yuka Kato, Yumi Hanazato, and Mikiko Sakaguchi for their contributions to animal experiments and Yuka Kawasawa-Imamura for meaningful advice on qPCR analyses.

Correspondence should be addressed to Sanae Hasegawa-Ishii at sanae_ ishii@ks.kyorin-u.ac.jp or Fumiaki Imamura at fui1@psu.edu.

https://doi.org/10.1523/ENEURO.0403-19.2020 Copyright (C) 2020 Hasegawa-Ishii et al.

This is an open-access article distributed under the terms of the Creative Commons Attribution 4.0 International license, which permits unrestricted use, distribution and reproduction in any medium provided that the original work is properly attributed. mechanism of EPL shrinkage and recovery from nasal inflammation-induced atrophy to better understand the roles of the $\mathrm{OB}$ and olfactory cortex in nasal inflammation-associated brain damage.

Previous studies also showed that intranasal LPS administration induced gliosis in the EPL, which may have contributed to EPL shrinkage (Hasegawa-Ishii et al., 2017, 2019). On the other hand, the loss of odor input as a result of OSN loss may also have contributed. Long-term odor deprivation via unilateral naris closure (NC) induces $\mathrm{OB}$ atrophy and decreases the volume of the EPL and granule cell layer (GCL), with no evidence of histologic changes in the OE (Henegar and Maruniak, 1991; Maruniak et al., 1989a,b, 1990). However, it is not known whether NC induces inflammatory responses and which part of the EPL is affected by odor deprivation. Therefore, we performed a detailed examination of the histologic and biochemical changes and inflammatory responses in the OB and OE in adult mice experiencing long-term odor deprivation and chronic nasal inflammation.

\section{Materials and Methods}

\section{Animals}

Eight-week-old male C57BL/6JJmsSlc mice (Sankyo lab) were used in this study. The mice were deeply anesthetized with isoflurane and intranasally administered 10- $\mu$ l physiologic saline or LPS from Escherichia coli 055:B5 $(1 \mathrm{mg} / \mathrm{ml}$; Sigma) three times per week for three, six, or 10 weeks (saline:3w, saline: $6 \mathrm{w}$, and saline:10w or LPS:3w, LPS:6w, and LPS:10w, respectively). Intranasal administrations were applied unilaterally to the left naris of each mouse, with the right side serving as an internal control. Another group of eight-week-old male mice underwent unilateral NC by brief cauterization and were housed for three, six, and 10 weeks (NC:3w, NC:6w, and NC:10w, respectively). For analyses of recovery, mice receiving LPS for 10 weeks were subsequently housed with no additional treatment for another two, six, and 10 weeks (LPS:10w+NT:2w, LPS:10w+NT:6w, and LPS:10w+NT:10w, respectively) or underwent NC (LPS: $10 w+N C: 10 w)$.

\section{Immunostaining}

Mice were anesthetized with ketamine $(100 \mathrm{mg} / \mathrm{kg}$ body weight) and xylazine $(10 \mathrm{mg} / \mathrm{kg})$ and transcardially perfused with PBS, and then with $4 \%$ paraformaldehyde in PBS. Their heads were removed and placed in the same fixative at $4^{\circ} \mathrm{C}$ overnight. The rostral half of the calvaria and the nasal bone were then placed en block in $2 \times \mathrm{K}-\mathrm{CX}$ (Falma) for $2.5 \mathrm{~h}$ for decalcification and then washed with water for $6 \mathrm{~h}$. The brains were cryoprotected with $30 \%$ sucrose in PBS (wt/vol) at room temperature overnight, embedded in OCT compound (Sakura Finetek USA Inc.), and maintained at $-80^{\circ} \mathrm{C}$ until use.

Olfactory tissues were coronally cut on a cryostat into $20 \mu \mathrm{m}$ slices, mounted on slide glasses, dried and stored at $-80^{\circ} \mathrm{C}$ until use. The sections were rehydrated with TBST $[10 \mathrm{mmol} / \mathrm{I}$ Tris- $\mathrm{HCl}(\mathrm{pH} 7.4)$ and $100 \mathrm{mmol} / \mathrm{I} \mathrm{NaCl}$ with $0.1 \%$ Tween 20], blocked with blocking buffer [1\% bovine serum albumin for immunohistochemistry or $5 \%$ 
Table 1: List of antibodies. Information of all antibodies used in this study is listed, including the name, host, dilution, source, and immunogen

\begin{tabular}{|c|c|c|c|c|}
\hline $\begin{array}{l}\text { Antibody } \\
\text { against }\end{array}$ & Host & Dilution & Source & Immunogen \\
\hline IL-1 $\beta$ & Goat & $1: 200$ & R\&D Systems & E. coli-derived recombinant mouse IL-1 $\beta / \mathrm{IL}-1 \mathrm{~F} 2$ Val118-Ser269 \\
\hline OMP & Goat & $1: 1000$ & $\begin{array}{l}\text { Fujifilm Wako Pure } \\
\text { Chemical Corp. }\end{array}$ & Rodent OMP \\
\hline Calretinin & Rabbit & $1: 500$ & NeoMarkers & Recombinant full-length mouse calretinin protein \\
\hline lba-1 & Rabbit & $1: 200$ & Wako & $\begin{array}{l}\text { A synthetic peptide corresponding to the lba-1 C-terminal sequence } \\
\text { (PTGPPAKKAISELP) }\end{array}$ \\
\hline GFAP & Rabbit & $1: 1000$ & Dako Agilent & GFAP isolated from cow spinal cord \\
\hline TH & Rabbit & $1: 500$ & Millipore & Denatured TH from rat pheochromocytoma \\
\hline
\end{tabular}

normal donkey serum (v/v) for immunofluorescence in TBST] at room temperature for $1 \mathrm{~h}$, and incubated with primary antibodies diluted in blocking buffer overnight. The antibodies used in the present study are listed in Table 1. Sections were incubated with host-matched secondary antibodies (ImmPress rabbit-IgG, goat-IgG, or ratIgG; Vector) at room temperature for $1 \mathrm{~h}$ and stained with ImmPACT SG (Vector). Nuclei were stained with nuclear fast red (Muto) for $1 \mathrm{~min}$. Slices were cleared and coverslipped with HSR (Sysmex). Sections were examined with an EDC Eclipse light microscope equipped with a digital camera control unit, DS-Fi2/DS-L3 (Nikon).

For double immunostaining with fluorescence, Alexa Fluor 568-conjugated or 488-conjugated donkey antispecies IgGs (Thermo Fisher Scientific) were used as secondary antibodies (1:300). Nuclei were counterstained with 4',6-diamidino-2-phenylindole (DAPI). The sections were coverslipped with fluorescence mounting medium (Dako Agilent) and imaged using a fluorescence microscope with structured illumination (BZ-X710; Keyence).

\section{Quantitative real-time RT-PCR assay}

Mice from saline:10w, LPS:10w, and NC:10w groups were decapitated with a guillotine, and their brains were removed and dissected along the midline. OBs ipsilateral (left side) and contralateral (right side) to the treatment were obtained on ice under a stereomicroscope, snap frozen in liquid nitrogen, and stored at $-80^{\circ} \mathrm{C}$ until use. Total RNA was isolated from the frozen OBs using a NucleoSpin RNA kit (Macherey-Nagel GmbH \& Co KG) according to the manufacturer's instructions. RNA quality was checked by examining absorbance values, and first-strand cDNA was synthesized from $1 \mu \mathrm{g}$ total RNA with 200-U SuperScript III reverse transcriptase (Thermo Fisher Scientific) in the appropriate buffer in the presence of random primers, deoxyribonucleotide triphosphate mix, dithiothreitol, and 40-U RNase OUT (Thermo Fisher Scientific). Reverse transcription was performed at $25^{\circ} \mathrm{C}$ for $10 \mathrm{~min}, 50^{\circ} \mathrm{C}$ for $60 \mathrm{~min}$, and $70^{\circ} \mathrm{C}$ for $15 \mathrm{~min}$. Real-time PCR was performed using TB Green Premix Ex Taq II (Takara Bio Inc.), according to the manufacturer's instructions, in a total volume of $25 \mu \mathrm{l}$ with a Thermal Cycler Dice Real Time System II (Takara Bio Inc.) and 40 cycles of $95^{\circ} \mathrm{C}$ for $5 \mathrm{~s}$ and $60^{\circ} \mathrm{C} 30 \mathrm{~s}$. The primer sequences are listed in Table 2. Transcript levels were normalized to those of Gapdh from corresponding samples. Data are expressed as relative fold change using the $2^{-\Delta \Delta C T}$ calculation method.

\section{Image analyses and morphometry}

All samples were randomly numbered so that the analyses were performed by an experimenter who was blind to sample identities.

Table 2: Primer sequences

\begin{tabular}{|c|c|c|c|}
\hline Target & Direction & Sequence $\left(5^{\prime} \rightarrow 3^{\prime}\right)$ & Accession no. \\
\hline IL-1 $\beta$ & Forward & CCTCACAAGCAGAGCACAA & NM_008361.4 \\
\hline $\operatorname{TNF} \alpha$ & Forward & CTGAGTTCTGCAAAGGGAGAG & NM_001278601.1 \\
\hline \multirow[t]{2}{*}{ IL-10 } & Forward & TTGAATTCCCTGGGTGAGAAG & NM_010548.2 \\
\hline & Reverse & TCCACTGCCTTGCTCTTATTT & \\
\hline Iba-1 & Forward & GACGTTCAGCTACTCTGACTTT & NM_019467.3 \\
\hline GFAP & Reverse & AGAGCAGTCACAGGGTAAGA & \\
\hline \multirow[t]{2}{*}{ GAPDH } & Forward & TCCTCAGTGTAGCCCAAGA & NM_001289726.1 \\
\hline & Reverse & GGAGAAACCTGCCAAGTATGA & \\
\hline
\end{tabular}

Information of all primers used in this study is listed, including the target, direction, sequence, and accession number. 


\section{Relative OB size}

After cryoprotection, the nasal bones over the OBs were carefully removed under the microscope (Stemi305; Carl Zeiss AG) and the dorsal views of the ipsilateral and contralateral OBs were imaged with a digital camera (DS-Fi2/DSL3; Nikon) attached to the microscope. Using these dorsal views, ipsilateral and contralateral $\mathrm{OB}$ areas were measured separately using Photoshop software (Adobe Systems Inc.). The number of pixels contained in the OB was measured and converted to area $\left(\mathrm{mm}^{2}\right)$, and the ratio of the ipsilateral $\mathrm{OB}$ area to the contralateral area (ipsilateral OB area/contralateral OB area) was calculated. Values were compared among saline: $3 \mathrm{w}$, saline: $6 \mathrm{w}$, and saline:10w ( $n=4,3$, and 4, respectively), LPS:3w, LPS:6w, and LPS:10w ( $n=4,3$, and 4, respectively); and NC:3w, NC:6w, and NC:10w ( $n=4,4$, and 5, respectively), and among LPS:10w, LPS:10w+NT:10w, and LPS:10w+NC:10w $(n=4)$ or LPS:10w, LPS:10w+NT:2w, LPS:10w+NT:6w, and LPS:10w+NT:10w $(n=4)$.

\section{OB layer areas}

Nuclei in coronal sections of the OBs were stained with nuclear fast red. The number of pixels enclosed by the boundaries of each layer was measured and converted to area $\left(\mathrm{mm}^{2}\right)$ using Photoshop software (Adobe Systems Inc.). As the rostral parts of the $\mathrm{OB}$ are much smaller and caudal parts have much thinner ONL, layer analyses were performed using the sections from the "middle OBs" that included 3-to 4- $\mathrm{mm}^{2}$ area of the whole $\mathrm{OB}$ and $\geq 0.5 \mathrm{~mm}^{2}$ area of the ONL on the contralateral side. Three these sections per mouse were selected for the statistical analysis. The ratio of each layer was determined by dividing the area from the ipsilateral side by the area from the contralateral side. Values were compared among saline:10w, LPS:10w, and NC:10w, or LPS:10w, LPS:10w+NT:10w, and LPS:10w+NC:10w $(n=3)$.

\section{sEPL area}

Sections from the middle OBs were stained with DAPI and for calretinin to label mitral cells, granule cells with synaptic connections with mitral cells, and some periglomerular cells. Within the EPL, the calretinin-positive area was regarded as the deep EPL (dEPL) and the calretininnegative area was regarded as the SEPL (Malz et al., 2000). The pixel numbers enclosed by the boundaries of sEPL were measured and converted to the area $\left(\mathrm{mm}^{2}\right)$ by using Photoshop software (Adobe Systems Inc.). The ratio of the SEPL was determined by dividing the area of the sEPL by the total EPL area. Values were compared among saline:10w, LPS:10w, and NC:10w or LPS:10w, LPS:10w+NT:10w, and LPS:10w+NC:10w $(n=3)$.

\section{Olfactory marker protein (OMP)-positive length}

Coronal sections of the OE were stained with DAPI and for the OMP expressed by mature OSNs. Two OE sections per each mouse that were $300-600 \mu \mathrm{m}$ rostral to the anterior tip of the OB were selected for the analysis. The length along the surface of the OE including the septal $\mathrm{OE}$, the first, second and third turbinates (total OE), and the length along the OMP-positive epithelium within the total OE were measured using ImageJ software. The ratio of OMP-positive length was determined by dividing the length of the OMP-positive epithelium by the total OE length. Values were compared among LPS:10w, LPS:10w+NT:10w, and LPS:10w+NC:10w $(n=3)$.

\section{Experimental design and statistical analysis}

Comparisons of the relative OB sizes among saline, LPS, and NC treatments were statistically analyzed by two-way analysis of variance (two main effects of treatment and time), followed by Tukey's HSD post hoc tests for multiple comparisons. A $p<0.05$ indicated a significant difference. The areas of each layer of the $O B$, ratios of sEPLs, transcript amounts, time courses of the changes in the relative OB size, and ratios of OMP-positive length to total OE length were statistically analyzed using Tukey's HSD tests. Statistical analyses were performed using Statistica software (Dell Software). Values are reported as means \pm SEMs.

\section{Results}

\section{OE inflammation and loss of OSNs}

Macrophages (F4/80 positive) and neutrophils (Ly-6G positive) did not infiltrate the $O E$ in animals administered saline (Fig. $1 A, D$ ) but accumulated in the $\mathrm{OE}$ and lamina propria, some of which expressed interleukin-1 $\beta$ (IL-1 $\beta$ ), of LPS:10w (Fig. 1B,E). Few if any macrophages or neutrophils were observed in OE of NC:10w (Fig. 1C,F), indicating that $\mathrm{NC}$ did not induce nasal inflammation.

In the saline:10w, mature (OMP positive) and immature [growth-associated protein 43 (GAP43) positive] OSNs were detected in the OE (Fig. 1G,J), whereas these were lost from the ipsilateral OE in LPS:10w (Fig. $1 H, K$ ), consistent with our previous study. OSNs were detected in the ipsilateral and contralateral OE in $\mathrm{NC}: 10 \mathrm{w}$ (Fig. 1/,L), indicating that 10 weeks of NC did not induce a loss of OSNs.

\section{Gross atrophy of the OB}

In saline-treated mice, ipsilateral and contralateral OBs were similar in size, because the ipsilateral areas were almost $100 \%$ relative to the contralateral OB areas at all time points examined $(97.6 \pm 3.3 \%, 98.6 \pm 2.5 \%$, and $98.7 \pm 2.4 \%$ of the contralateral values in saline:3w, saline:6w, and saline:10w, respectively; Fig. $2 A, D)$, indicating that the administration procedure did not induce atrophy. In LPS-treated mice, the ipsilateral OB areas gradually decreased to $81.0 \pm 2.5 \%$ and $77.2 \pm 1.9 \%$ of the contralateral values at six and 10 weeks, respectively, which were significantly lower than in saline-treated mice at the same time points (Fig. 2B,D). In NC mice, the areas of ipsilateral OB significantly decreased to $81.1 \pm 1.8 \%$ of the contralateral values at 10 weeks (Fig. $2 C, D$ ). The contralateral OB sizes did not significantly differ among saline-treated, LPS-treated, and NC mice (data not shown). These results indicate that LPS and NC treatments similarly induced atrophy of the ipsilateral $\mathrm{OB}$ within 10 weeks.

\section{Specific layer shrinkage}

To examine which layers of the OB contributed to the observed atrophy, we measured the area of each layer 

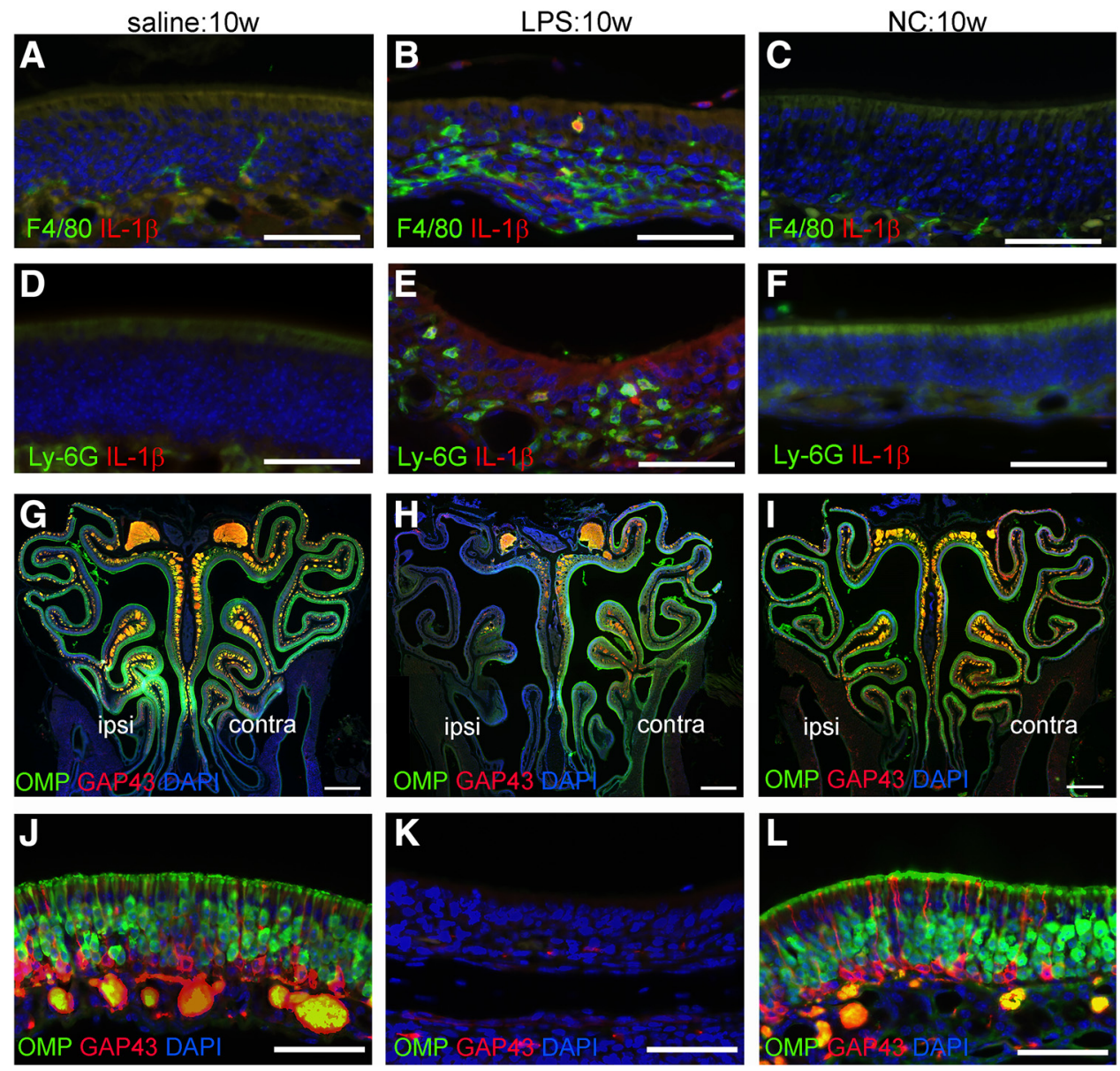

Figure 1. Nasal inflammation and loss of OSNs. $\boldsymbol{A}-\boldsymbol{C}$, Immunofluorescence for F4/80 (green), IL-1 $\beta$ (red), and nuclei (DAPI, blue) in the ipsilateral OE in saline:10w $(\boldsymbol{A})$, LPS:10w $(\boldsymbol{B})$ and NC:10w $(\boldsymbol{C})$. F4/80-immunopositive macrophages infiltrated the OE in LPS:10w $(\boldsymbol{B})$ but not in saline:10w $(\boldsymbol{A})$ or NC:10w $(\boldsymbol{C})$. Some F4/80-immunopositive macrophages in the OE expressed IL-1 $\beta$ in LPS:10w (B). $\boldsymbol{D}-\boldsymbol{F}$, Immunofluorescence for Ly-6G (green), IL-1 $\beta$ (red), and nuclei (DAPI, blue) in the ipsilateral OE in saline:10w $(\boldsymbol{D})$, LPS:10w $(\boldsymbol{E})$, and NC:10w $(\boldsymbol{F})$. Ly-6G-immunopositive neutrophils infiltrated the OE in LPS:10w $(\boldsymbol{E})$ but not in saline:10w $(\boldsymbol{D})$ or NC:10w $(\boldsymbol{F})$. A few Ly-6G-immuopositive neutrophils in the OE expressed IL-1 $\beta$ in LPS:10w $(\boldsymbol{E})$. G-I, Coronal sections of OE stained for OMP (green), GAP43 (red), and nuclei (DAPI; blue) in saline:10w (G), LPS:10w (H), and NC:10w (I). OMP- and GAP43immunopositive mature and immature OSNs were lost in the ipsilateral OE in LPS:10w $(\boldsymbol{H})$ but not in saline:10w (G) or NC:10w (I). $\boldsymbol{J}-\boldsymbol{L}$, Magnified views of ipsilateral OE stained for OMP (green), GAP43 (red), and nuclei (DAPI, blue). Scale bars: $50 \mu \mathrm{m}(\boldsymbol{A}-\boldsymbol{F}, \boldsymbol{J}-\boldsymbol{L}$ ) and $500 \mu \mathrm{m}(\mathbf{G}-\mathbf{I})$.

using coronal sections from the middle of the ipsilateral and contralateral OBs (Fig. 3A-D). These measurements confirmed that saline treatment did not reduce the area of the whole OB [sum of ONL, GL, EPL, mitral cell layer (MCL) plus the internal plexiform layer (IPL; MCL+IPL) and GCL; $101.6 \pm 1.1 \%$ of contralateral values], whereas ipsilateral areas were significantly lower both in LPS: $10 \mathrm{w}$ $(74.1 \pm 2.3 \%)$ and NC:10w (82.2 $\pm 3.2 \%$; Fig. $3 E)$. In LPS: $10 \mathrm{w}$, the areas of the ipsilateral ONL, GL, and EPL relative to their contralateral counterparts $(36.6 \pm 5.4 \%$, $59.3 \pm 1.5 \%$, and $83.7 \pm 1.1 \%$, respectively) were significantly lower than in the saline:10w $(97.0 \pm 10.2 \%, 105.4$ $\pm 4.5 \%$, and $105.0 \pm 3.3 \%$, respectively; Fig. $3 E$ ). The ratios for MCL+IPL and GCL did not significantly change in the LPS:10w compared with those in the saline-treated controls. Whereas NC did not alter the ratios for ONL, those for GL, EPL, MCL+IPL, and GCL $(83.6 \pm 6.0 \%$, $71.9 \pm 2.3 \%, 79.8 \pm 2.5 \%$, and $82.8 \pm 4.2 \%$, respectively) were significantly lower than in salinetreated controls $(105.4 \pm 4.5 \%, 105.0 \pm 3.3 \%, 105.1 \pm$ $5.4 \%$, and $101.4 \pm 6.3 \%$, respectively; Fig. $3 E$ ). Thus, different layers contributed to the $\mathrm{OB}$ atrophy observed in LPS-treated and NC mice: LPS induced shrinkage of ONL, GL, and EPL, and NC induced shrinkage of GL, EPL, MCL+IPL, and GCL (Fig. 3E).

We confirmed the shrinkage of ONL and GL by immunohistochemistry for OMP, a marker for mature OSNs. The ONL and GL were immunopositive for OMP in salinetreated controls (Fig. $4 A, D$ ), whereas the staining in the lateral ONL of the ipsilateral OB was almost completely lost in LPS:10w (Fig. 4B,E). The number and the size of glomeruli were remarkably decreased in the lateral side of the ipsilateral OB, whereas the medial side was largely intact in LPS:10w. Immunoreactivity in ONL in the NC:10w (Fig. 4C,F) was similar to that in the saline:10w, indicating that the OSNs were histologically intact in NC: $10 \mathrm{w}$. 

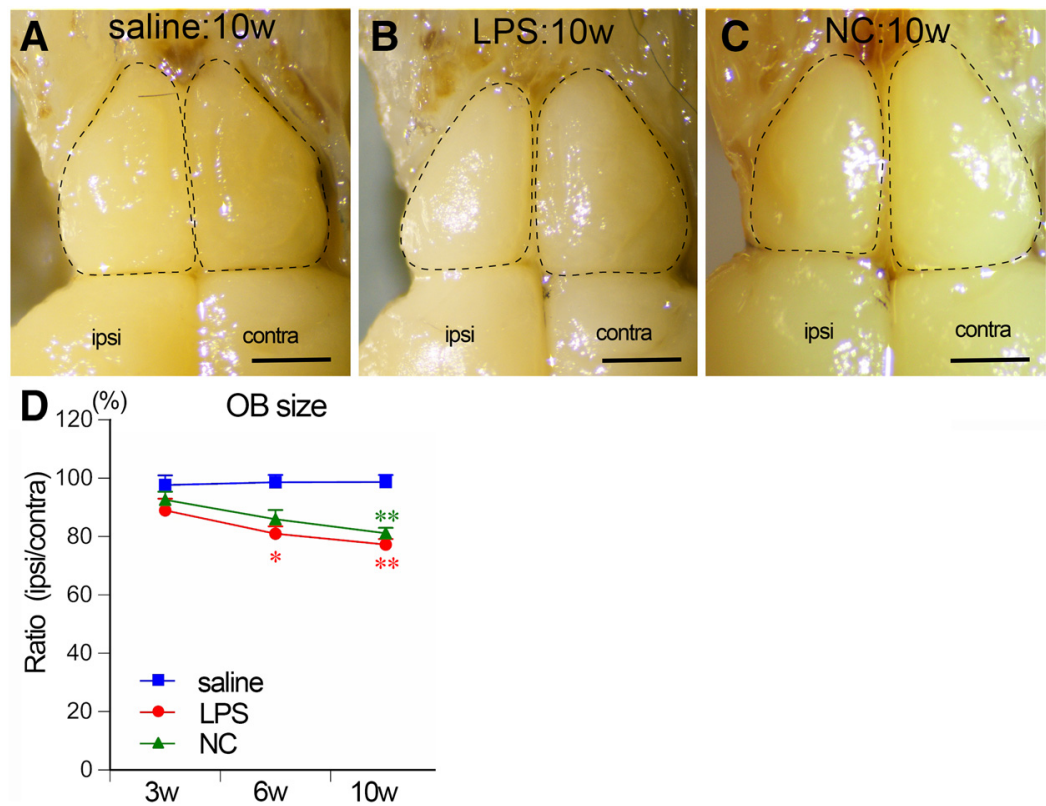

Figure 2. Atrophy of the OB. $\boldsymbol{A}-\boldsymbol{C}$, Dorsal views of the OBs of saline:10w $(\boldsymbol{A})$, LPS:10w $(\boldsymbol{B})$, and NC:10w $(\boldsymbol{C})$. Ipsilateral OBs atrophied in LPS:10w $(\boldsymbol{B})$ and NC:10w $(\boldsymbol{C})$. Scale bars: $1 \mathrm{~mm}$. $\boldsymbol{D}$, Graph presents the time course of changes in the OB size. Ratios of ipsilateral OB areas to the contralateral ones gradually decreased in LPS:10w and NC:10w; $* p<0.05, * * p<0.01$ versus saline:10w.

A subpopulation of juxtaglomerular cells that extend their dendrites into glomeruli expresses tyrosine hydroxylase (TH) in an activity-dependent manner (Cho et al., 1996), such that TH immunostaining is detected in the GL in saline-treated controls (Fig. 4G). TH expression in the GL was weaker in LPS:10w than in saline:10w, but was much weaker in NC:10w (Fig. 4H,I).

\section{Thinning of the sEPL}

We further characterized the shrinkage of the EPL after 10 weeks of LPS and NC to determine which sublayer was more vulnerable to nasal inflammation and odor deprivation. The sEPL can be distinguished from the dEPL by its weaker calretinin immunostaining, which is expressed by mitral cells and connecting granule cells (Fig.

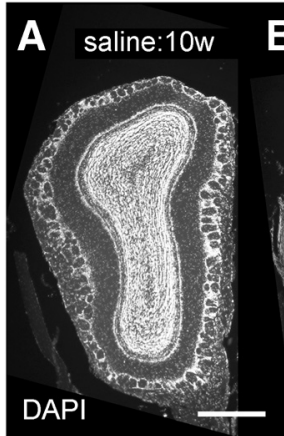

\section{B LPS:10w C NC:10w}

E
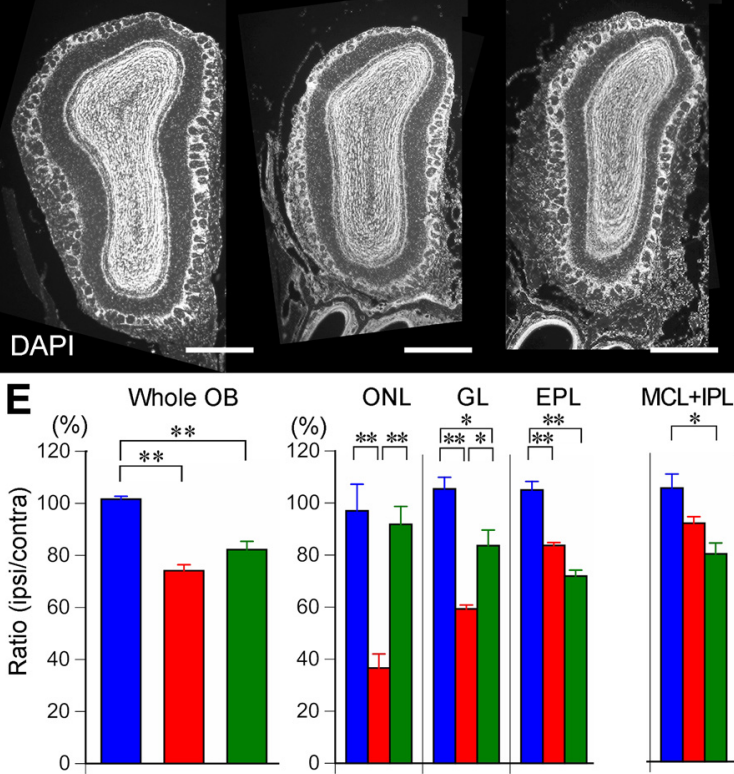

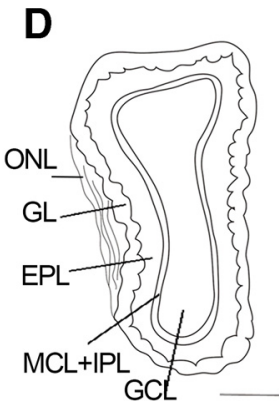

GCL

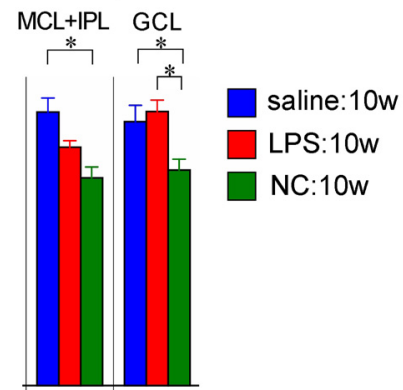

Figure 3. Shrinkage of layers in the OB. $\boldsymbol{A}-\boldsymbol{C}$, Coronal sections of the ipsilateral OBs stained with DAPI. $\boldsymbol{D}$, Schematic image of the $\mathrm{OB}$ representing each layer. $\boldsymbol{E}$, Graphs present the ratios of the ipsilateral layer areas to the contralateral ones. Whole OB contains all layers. ONL, GL, and EPL shrank in the LPS:10w, whereas GL, EPL, MCL+IPL, and GCL shrank in the NC:10w; $* p<0.05$, $* * p<0.01$. Scale bars: $500 \mu \mathrm{m}(\boldsymbol{A}-\boldsymbol{D})$. 


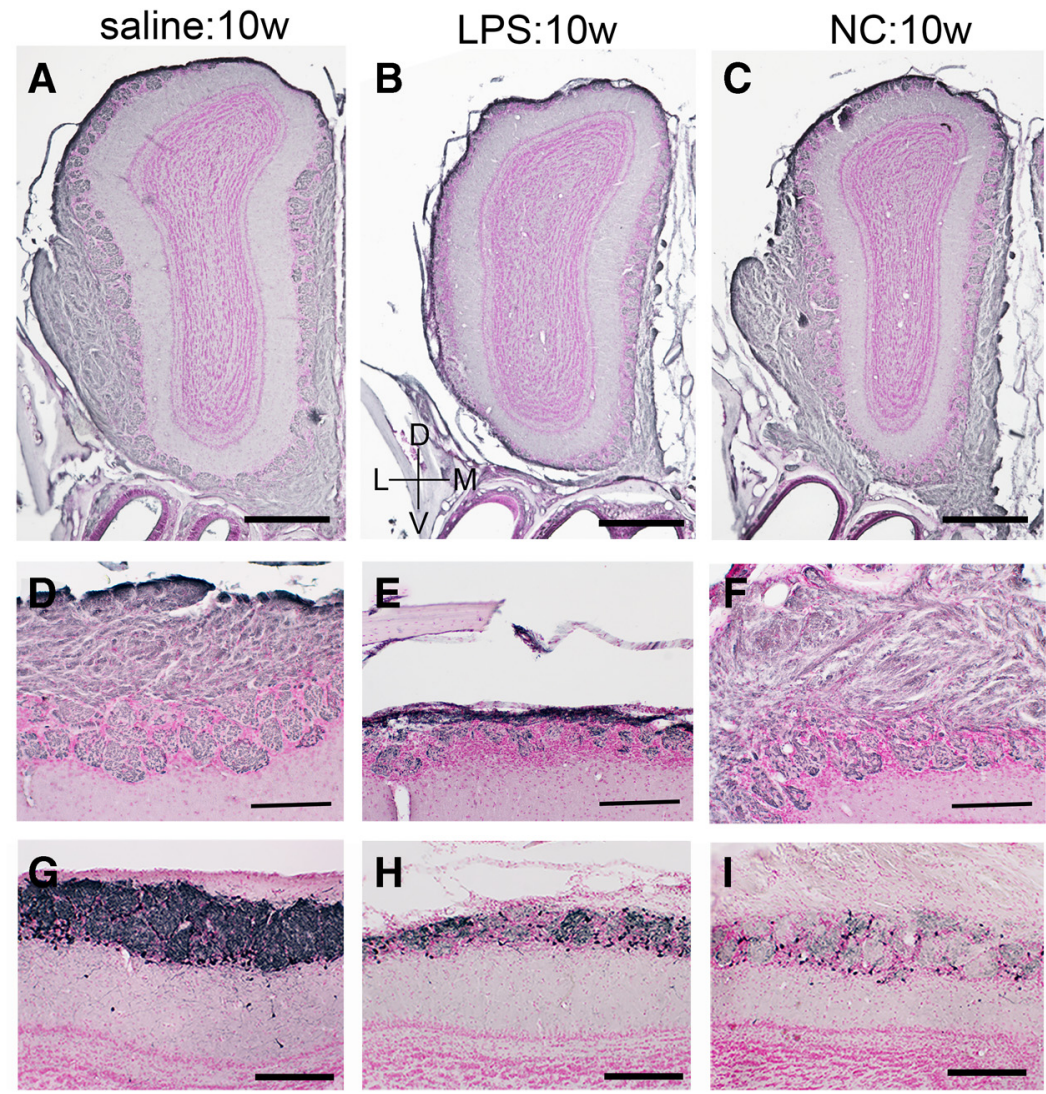

Figure 4. OMP and TH expression in the OB. $\boldsymbol{A}-\boldsymbol{F}$, Immunohistochemistry for OMP, representing ONL and GL, in ipsilateral OBs from saline:10w $(\boldsymbol{A}, \boldsymbol{D})$, LPS:10w $(\boldsymbol{B}, \boldsymbol{E})$, and NC:10w $(\boldsymbol{C}, \boldsymbol{F})$. OMP-immunopositive axon terminals of OSNs were lost particularly in the lateral side of the ipsilateral OB in LPS:10w $(\boldsymbol{B}, \boldsymbol{E})$ but not in saline:10w $(\boldsymbol{A}, \boldsymbol{D})$ or NC:10w $(\boldsymbol{C}, \boldsymbol{F})$. $\boldsymbol{D}-\boldsymbol{F}$, Magnified views of lateral side of the OBs in $\boldsymbol{A}-\boldsymbol{C}$. G-I, Immunohistochemistry for TH expressed by some juxtaglomerular cells. TH expression decreased in LPS:10w $(\boldsymbol{H})$ and in NC:10w $(\boldsymbol{I})$ compared with that in the saline:10w $(\boldsymbol{G})$. Nuclei were stained with nuclear fast red. Scale bars: $500 \mu \mathrm{m}(\boldsymbol{A}-\boldsymbol{C})$ and $200 \mu \mathrm{m}(\boldsymbol{D}-\boldsymbol{I})$.

$5 A-F$; Malz et al., 2000). In saline-treated controls, the sEPL was almost $50 \%$ of the total EPL in ipsilateral and contralateral OBs (Fig. $5 A, D, G$ ). By contrast, this was reduced to $38.0 \%$ (Fig. 5B,E,G) and $30.5 \%$ (Fig. 5C,F,G) in the ipsilateral OBs after 10 weeks of LPS and NC, respectively. Thus, the superficial layer of the EPL is affected by inflammation and odor deprivation, with greater sensitivity to odor deprivation.

\section{Glial responses and cytokine expression}

Microglia have a small cell body and thin radially projecting processes, and are distributed evenly throughout the brain and $O B$ under normal conditions, as seen in salinetreated control (Fig. 6A,D). In LPS:10w, microglia were activated with larger cell bodies and thicker processes, particularly on the lateral side of the ipsilateral OB (Fig. 6B,E). Quantitative PCR analysis revealed that the amount of transcript for ionized calcium-binding adaptor molecule-1 (lba-1) was $\sim 2.5$ times higher in the ipsilateral OBs of LPS:10w than in the saline controls (Fig. 6G). By contrast, microglia appeared normal, and the Iba-1 transcript amount did not change in the NC:10w (Fig. 6C,F,G).

Astrocytes were located mainly in the GL, SEPL and GCL of saline-treated (Fig. $7 A, D$ ) and NC-treated mice
(Fig. 7C,F). However, hypertrophic astrocytes were distributed throughout the EPL as well as the GL in LPS:10w (Fig. 7B,E). Quantitative PCR analysis revealed that the amounts of transcript for glial fibrillary acidic protein (GFAP) significantly increased in LPS:10w and NC:10w (Fig. 7G), suggesting that astrocytes reacted to NC, although their morphology was not prominently altered.

As we observed robust glial activation in the ipsilateral OBs of LPS:10w, we expected to observe another sign of neuroinflammation. We performed quantitative PCR to measure the expression of IL- $1 \beta, \mathrm{TNF} \alpha$, IL- 6 , and COX-2 as representative proinflammatory factors and of IL-10, $\operatorname{TGF} \beta$, and BDNF as representative anti-inflammatory factors. In the ipsilateral OBs, 4.4 times more IL- $1 \beta$ and 5.8 times more TNF $\alpha$ were expressed in the LPS:10w than in the saline controls $(p=0.051$ for $\mathrm{IL}-1 \beta$ and $p<0.01$ for $\operatorname{TNF} \alpha$ ), with no change detected in the NC:10w (Fig. $7 H, I)$. We also found that IL-10 expression was six and three times higher in the LPS:10w and NC:10w, respectively (Fig. $7 \mathrm{~J}$ ). There were no changes in the expression levels of other inflammatory factors examined with LPS or NC treatment. These results indicate that 10 weeks of LPS administration, but not NC, induces neuroinflammation in the OB. 

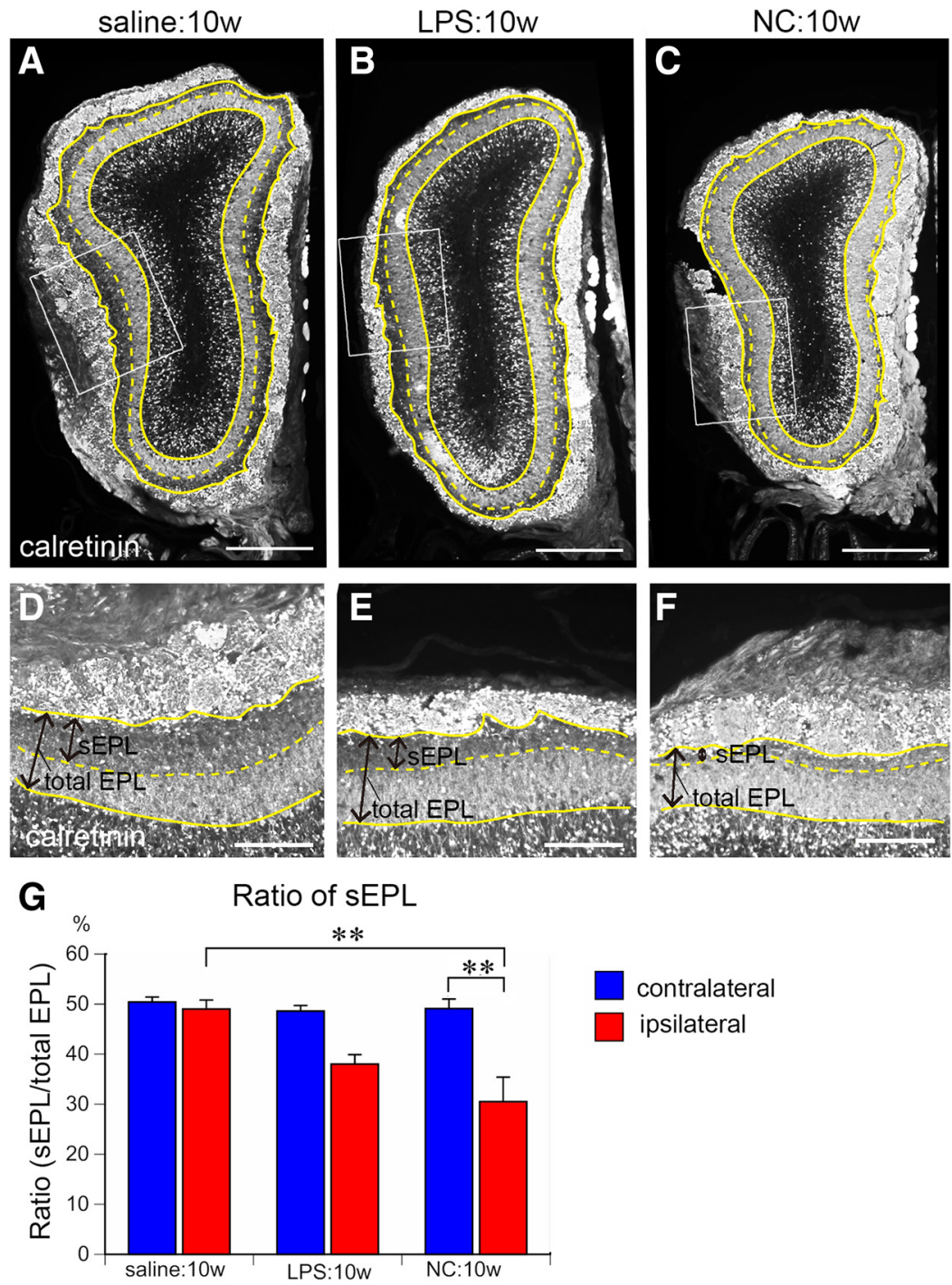

Figure 5. Shrinkage of SEPL. $\boldsymbol{A}-\boldsymbol{F}$, Coronal sections of the ipsilateral OB stained for calretinin (expressed by mitral cells and some granule cells connecting to mitral cells in the dEPL). Solid yellow lines represent the borders of the GL and EPL or EPL and MCL. Dotted yellow lines represent the borders of SEPL and dEPL. $\boldsymbol{D}-\boldsymbol{F}$, Magnified views of areas indicated by rectangles in $\boldsymbol{A}-\boldsymbol{C}$. The sEPL preferentially shrank in LPS:10w and NC:10w. G, Graph presents the ratios of sEPL to total EPL. The ratios of sEPL to total EPL decreased in ipsilateral EPLs in LPS:10w and NC:10w; $* * p<0.01$. Scale bars: $500 \mu \mathrm{m}(\boldsymbol{A}-\boldsymbol{C})$ and $200 \mu \mathrm{m}$ $(D-F)$.

\section{Remission of nasal inflammation and neuroinflammation}

The OB recovers from LPS-induced atrophy once nasal inflammation subsides (Hasegawa-Ishii et al., 2019). In the present study, we examined whether odor deprivation is related to OB recovery.

First, we examined whether nasal inflammation subsides in the presence (LPS:10w+NT:10w) or absence (LPS:10w+NC:10w) of odor input. Very few F4/80-positive macrophages or Ly-6G-positive neutrophils were detected in the OE in the LPS:10w+NT:10w and LPS: $10 w+N C: 10 w$ (data not shown), indicating that nasal inflammation subsided regardless of odor input. To examine the recovery of OSNs, we calculated the OMP-positive length relative to the entire OE length. In LPS:10w, $18.9 \pm$ $5.5 \%$ of OE length was OMP positive (Fig. $8 A, D$ ), which increased to $36.9 \pm 3.3 \%$ in the LPS: $10 \mathrm{w}+\mathrm{NT}: 10 \mathrm{w}$ and significantly increased to $46.6 \pm 3.7 \%$ in the LPS:10w+NC:10w (Fig. 8B-D). These results indicate that OSNs were able to recover from inflammation-induced damage in the presence and absence of odor input.

We also examined neuroinflammation in the $\mathrm{OB}$ during this recovery period by assessing glial activation. Compared with that in the LPS:10w, the size, numbers, and morphology of microglia returned to normal in both the LPS:10w+NT:10w and LPS:10w+NC:10w (Fig. 8E-G). Similarly, the hypertrophy and accumulation of astrocytes in the EPL in the LPS:10w were reversed in the LPS:10w+NT:10w and LPS:10w+NC:10w (data not shown). These results indicate that neuroinflammation subsided in the $\mathrm{OB}$ in the presence or absence of odor input. 

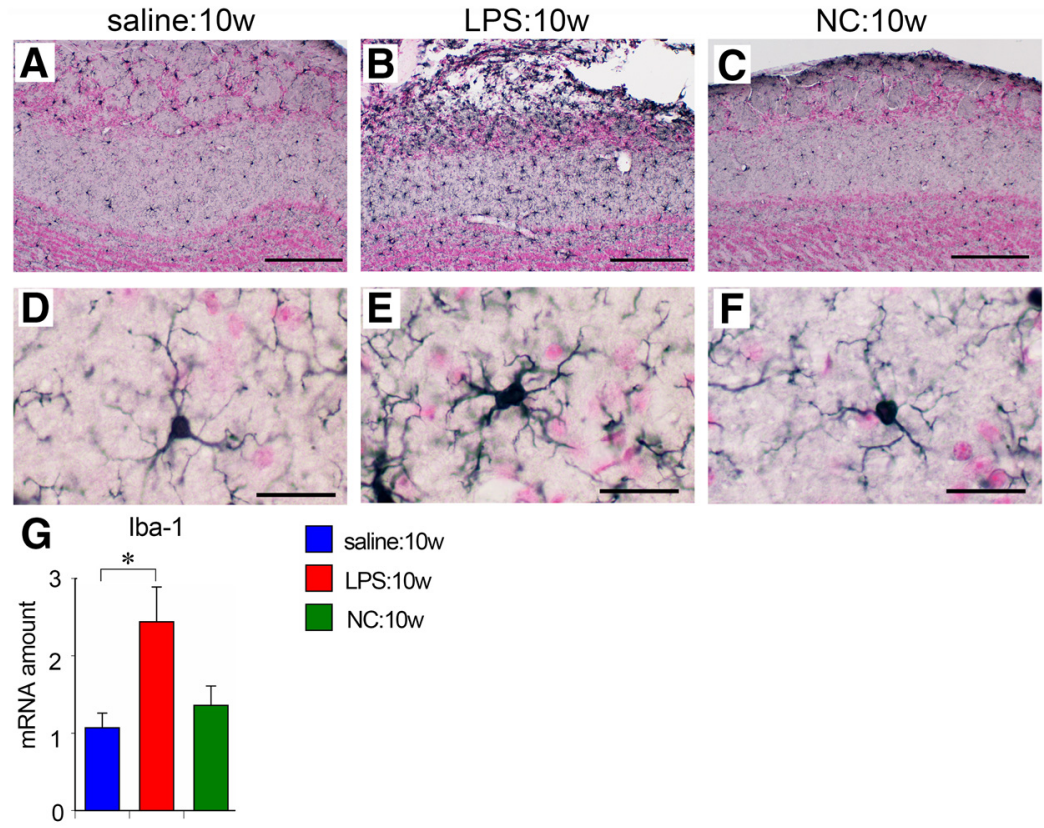

saline:10w

LPS:10w

NC:10w

Figure 6. Microglial activation in the OB. $\boldsymbol{A}-\boldsymbol{F}$, Immunohistochemistry for Iba-1 in ipsilateral OBs. Microglia were activated in LPS:10w $(\boldsymbol{B}, \boldsymbol{E})$ but not in NC:10w $(\boldsymbol{C}, \boldsymbol{F})$ compared with those in the saline:10w $(\boldsymbol{A}, \boldsymbol{D})$. Representative microglia are magnified in $\boldsymbol{D}-$ $\boldsymbol{F}$. Nuclei were stained for nuclear fast red. Scale bars: $200 \mu \mathrm{m}(\boldsymbol{A}-\boldsymbol{C})$ and $20 \mu \mathrm{m}(\boldsymbol{D}-\boldsymbol{F})$. $\boldsymbol{G}$, Graph presents the relative amounts of transcript for lba-1 in ipsilateral OBs. The lba-1 transcript amount in the LPS:10w was $\sim 2.5$ times than in the saline:10w; $* p<0.05$.

\section{Recovery from OB atrophy}

We next examined whether the atrophy of the OB recovers in the presence or absence of odor input during the recovery phase. The average size of the ipsilateral
OB significantly increased from $77.2 \pm 1.9 \%$ of the contralateral side in LPS: $10 \mathrm{w}$ to $92.0 \pm 4.3 \%$ in the LPS:10w+NT:10w (Fig. 9A,B,D). The areas of OB gradually increased during 10 weeks of nontreatment (Fig. 9E). By
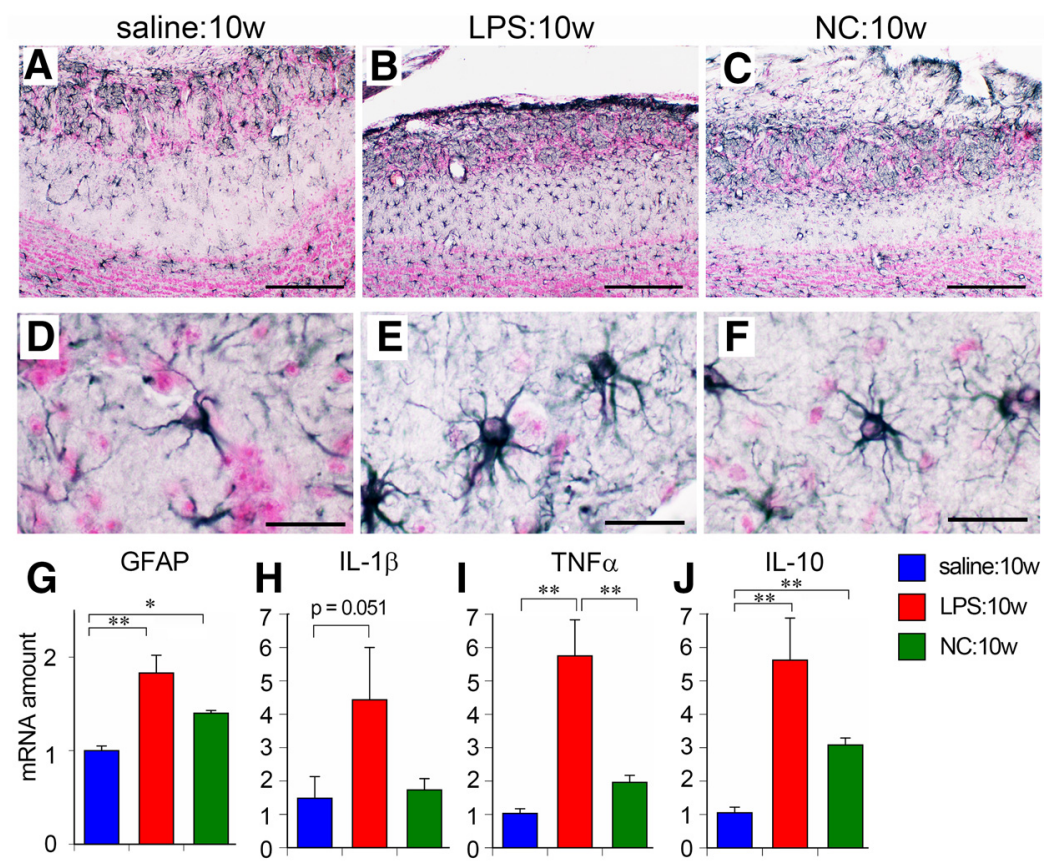

saline: $10 \mathrm{w}$

$\square$ LPS:10w

Figure 7. Astrocytic hypertrophy and neuroinflammation in the OB. $\boldsymbol{A}-\boldsymbol{F}$, Immunohistochemistry for GFAP in ipsilateral OBs. Astrocytes were hypertrophic in LPS:10w $(\boldsymbol{B}, \boldsymbol{E})$ but not prominently in NC:10w $(\boldsymbol{C}, \boldsymbol{F})$ compared with those in the saline:10w $(\boldsymbol{A}, \boldsymbol{D})$. Representative astrocytes are magnified in $\boldsymbol{D}-\boldsymbol{F}$. Nuclei were stained for nuclear fast red. Scale bars: $200 \mu \mathrm{m}(\boldsymbol{A}-\boldsymbol{C})$ and $20 \mu \mathrm{m}(\boldsymbol{D}-$ $\boldsymbol{F}$ ). G-J, Graphs present the relative amounts of transcript for GFAP, IL-1 $\beta$, TNF $\alpha$, and IL-10 in ipsilateral OBs. The transcript amounts of these molecules were significantly higher in the LPS:10w than in the saline:10w. The transcript amount of IL-10 was also higher in NC:10w than in saline:10w; $* p<0.05, * * p<0.01$. 

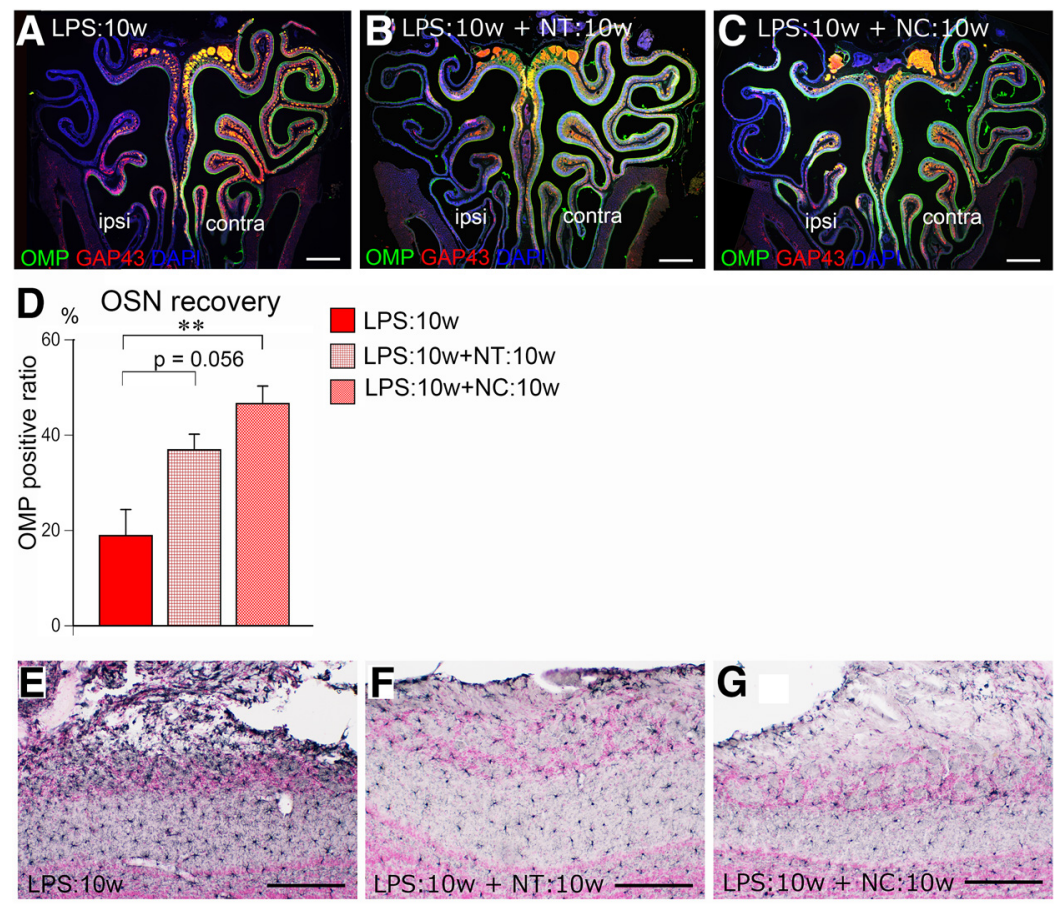

Figure 8. Remission of nasal-inflammation and neuro-inflammation. $\boldsymbol{A}-\boldsymbol{C}$, Coronal sections of the OE stained for OMP (green), GAP43 (red), and nuclei (DAPI, blue). OMP- and GAP43-immunopositive mature and immature OSNs are lost in LPS:10w (A) and regenerated in a patchy manner in the presence $(\boldsymbol{B})$ and absence $(\boldsymbol{C})$ of odor input. $\boldsymbol{D}$, Graph presents the OMP-immunopositive length relative to the total length of ipsilateral $\mathrm{OE}$. The ratio for the OMP-immunopositive length increased in the presence (LPS:10w+NT:10w) or absence (LPS:10w+NC:10w) of odor input; $* * p<0.01$. E-G, Immunohistochemistry for lba-1 in the ipsilateral OB. Microglia were activated in LPS:10w $(\boldsymbol{E})$ but returned to normal in LPS:10w+NT:10w $(\boldsymbol{F})$ and in LPS:10w+NC:10w $(\boldsymbol{G})$. Nuclei were stained with nuclear fast red. Scale bars: $500 \mu \mathrm{m}(\boldsymbol{A}-\boldsymbol{C})$ and $200 \mu \mathrm{m}(\boldsymbol{E}-\boldsymbol{G})$. In the OEs of NC:3w and NC:10w, there are more calretinin-immunopositive OSNs on the closed side than on the open side. Similarly, there are more calretinin-immunopositive OSNs in the OE of LPS:10w+NC:10w than in LPS:10w+NT:10w (Extended Data Fig. 8-1).

contrast, the OB size did not recover in the absence of odor input, because the average ipsilateral OB was $72.1 \pm 1.2 \%$ of the contralateral size in the LPS:10w+NC:10w (Fig. 9C,D).

To determine whether the recovery was layer specific, we compared the areas of each layer in coronal sections of the OBs among the LPS:10w, LPS:10w+NT:10w, and LPS:10w+NC:10w (Fig. 10A-C). The ONL was slightly but not significantly thicker in the LPS:10w+NT:10w and LPS: $10 \mathrm{w}+\mathrm{NC}: 10 \mathrm{w}$, at $58.4 \pm 2.5 \%$, and $59.2 \pm 9.6 \%$ of the contralateral layers, respectively (Fig. 10D). However, the areas of the GL and EPL significantly increased to $95.5 \pm 2.7 \%$ and $106.3 \pm 4.2 \%$, respectively, in the LPS: $10 \mathrm{w}+\mathrm{NT}: 10 \mathrm{w}$, as expected on the basis of a previous study. By contrast, GL was not different in the LPS:10w+NC:10w compared with that in the LPS:10w (Fig. $10 D$ ), whereas the EPL further decreased to $61.7 \pm 2.3 \%$ of that of the contralateral side (Fig. 10D). Moreover, the $M C L+I P L$ and GCL, which were not decreased in the LPS:10w, decreased in the LPS:10w+NC:10w compared with those in the LPS:10w and LPS:10w+NT:10w (Fig. 10D), suggesting that LPS:10w+NC:10w mice suffered from tandem insults of LPS and NC. These results indicate that the ONL recovered in the presence and absence of odor input, but the GL and EPL needed odor input for recovery.

The recovery of the ONL and GL in the LPS:10w+ NT:10w and LPS:10w+NC:10w was confirmed by immunohistochemistry for OMP (Fig. 11A-C). In addition, TH expression was restored in the LPS:10w+NT:10w, but not in the LPS:10w+NC:10w (Fig. 11D-F).

The reduced area of the SEPL in the ipsilateral OBs in the LPS:10w (Figs. 5B,E,G, 12A,D,G) recovered to $46.8 \%$ of the total EPL in the LPS:10w+NT:10w, which was similar to that of the contralateral OB (Fig. 12B,E,G). By contrast, the area of the sEPL further decreased to $23.9 \%$ of the total EPL in the ipsilateral OBs of the LPS:10w+ NC:10w, whereas the ratio did not change in the contralateral OBs (Fig. 12C,F,G).

\section{Discussion}

Chronic nasal inflammation induces a loss of OSNs and results in neuroinflammation and gross atrophy of the $\mathrm{OB}$ (Hasegawa-Ishii et al., 2019). The results from the present study extended these findings and demonstrated that chronic nasal inflammation and long-term odor deprivation have differential effects. LPS-induced chronic nasal inflammation caused shrinkage of superficial $O B$ layers (ONL, GL, and EPL) contributing to OB atrophy, whereas NC-induced long-term odor deprivation caused shrinkage of all layers except ONL (GL, EPL, MCL+IPL, and GCL), resulting in atrophy without inflammation or OSN loss.

The shrinkage of the ONL and GL in LPS-treated mice was attributed to the retraction of the axons of OSNs. The 

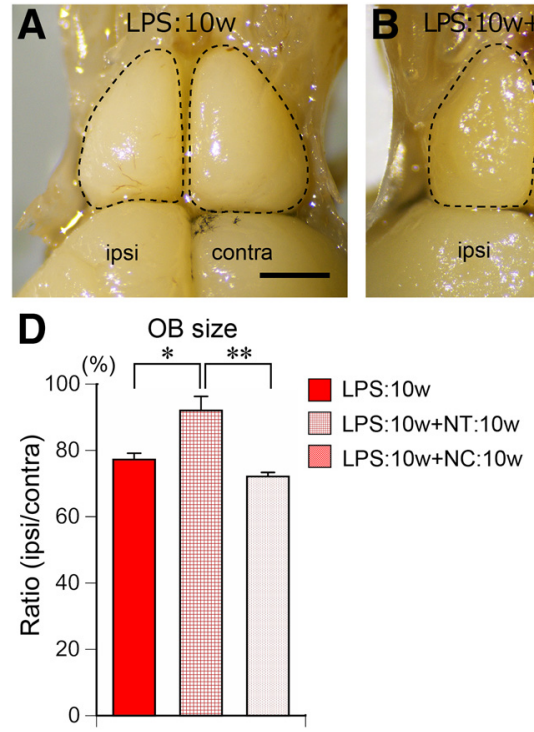

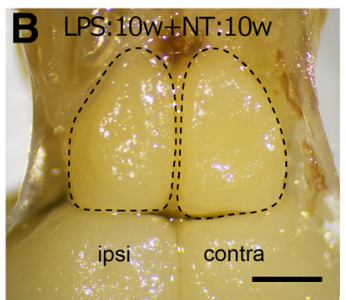

E

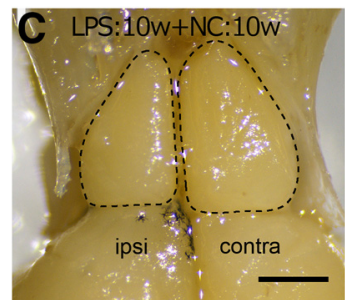

OB size

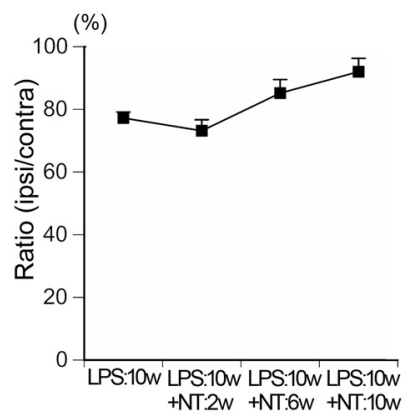

Figure 9. Recovery of $\mathrm{OB}$ in the presence or absence of odor input. $\boldsymbol{A}-\boldsymbol{C}$, Dorsal views of the OBs from LPS:10w (A), LPS:10w+NT:10w $(\boldsymbol{B})$, and LPS:10w+NC:10w $(\boldsymbol{C})$. Ipsilateral OB recovered from atrophy in the presence $(\boldsymbol{B})$ but not in the absence $(\boldsymbol{C})$ of odor input. Scale bars: $1 \mathrm{~mm}$. $\boldsymbol{D}$, Graph presents the ratios of the ipsilateral OB areas to the contralateral ones. The ratio significantly increased in the presence (LPS:10w+NT:10w) but not in the absence (LPS:10w+NC:10w) of odor input; $* p<0.05$, $* * p<0.01$. $\boldsymbol{E}$, Graph presents the time course of the ratio of the ipsilateral OB area to the contralateral one after 10 weeks of LPS administration.

shrinkage of the GL was also due to the retraction of dendrites of $\mathrm{OB}$ projection neurons in LPS-treated and NC mice. By contrast, the shrinkage of the GCL after 10 weeks of odor deprivation may have resulted from reduced neurogenesis of OB interneurons and/or survival of newly generated granule cells (Yamaguchi and Mori, 2005), processes that are facilitated by odor enrichment (Woo et al., 2006). Although the ONL, GL, and GCL were
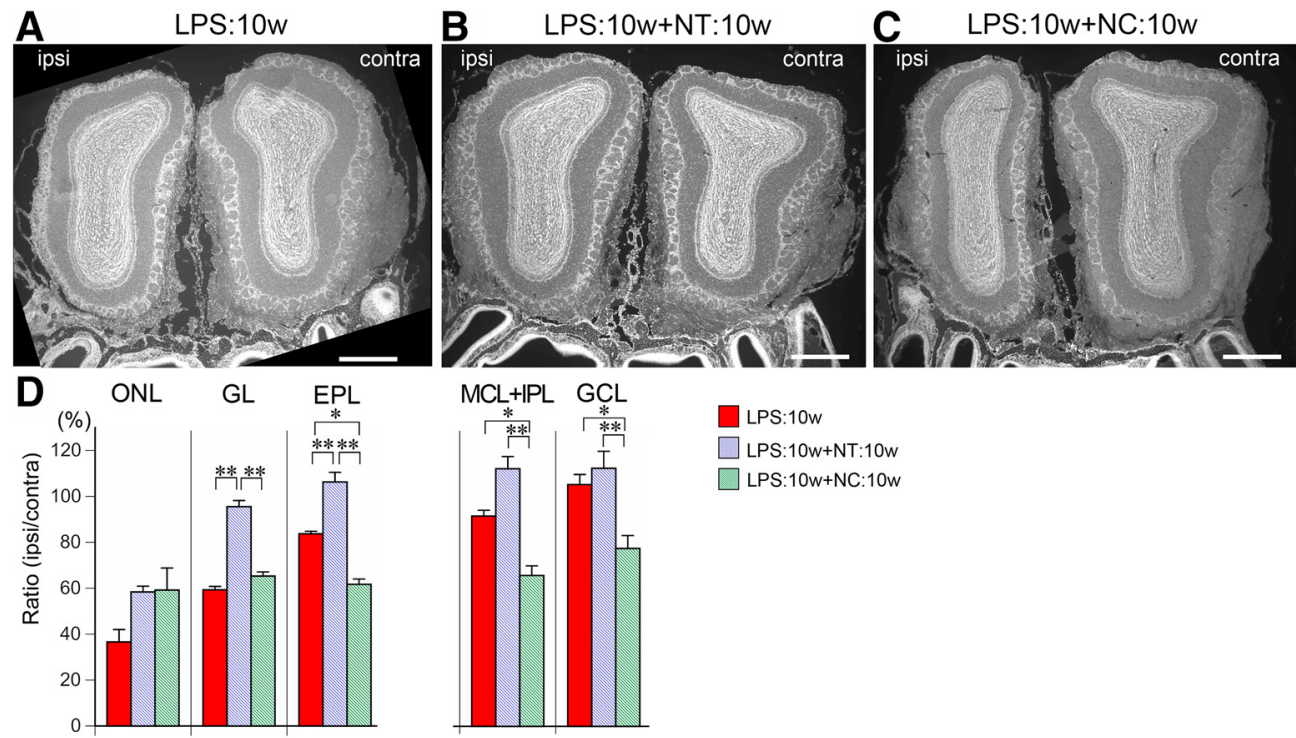

Figure 10. Recovery of each layer in the presence or absence of odor input. $\boldsymbol{A}-\boldsymbol{C}$, Coronal sections of the OBs stained with DAPI from LPS:10w $(\boldsymbol{A})$, LPS:10w+NT:10w $(\boldsymbol{B})$, and LPS:10w+NC:10w $(\boldsymbol{C})$. Ipsilateral OBs recovered from atrophy in the presence $(\boldsymbol{B})$ but not in the absence $(\boldsymbol{C})$ of odor input. Ipsilateral OBs further shrank in the absence of odor input (C). Scale bars: $500 \mu \mathrm{m}$. $\boldsymbol{D}$, Graphs present the ratios of the ipsilateral layer areas to the contralateral ones. ONL areas did not change among LPS:10w, LPS:10w+NT:10w, and LPS:10w+NC:10w, while GL and EPL, which shrank in LPS:10w, recovered in the presence (LPS:10w+NT:10w) but not in the absence (LPS:10w+NC:10w) of odor input. EPL area further shrank in the absence of odor input (LPS:10w+NC:10w). MCL+IPL and GCL, which did not shrink in LPS:10w, shrank in the absence (LPS:10w+NC:10w) but not in the presence (LPS:10w+NT:10w) of odor input; $* p<0.05, * * p<0.01$. 


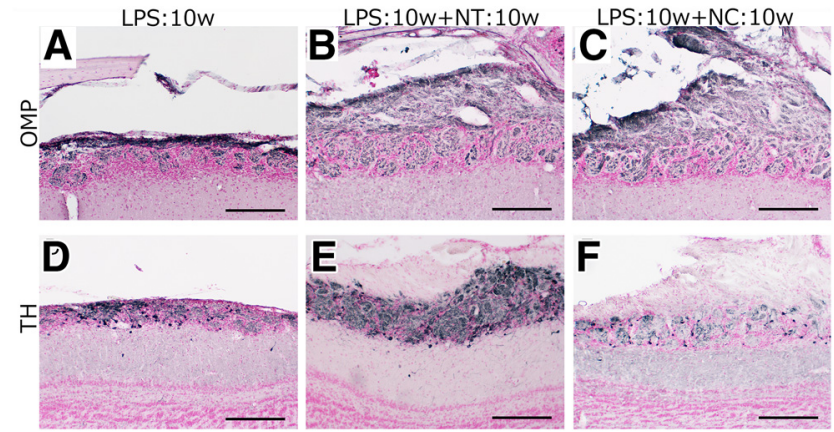

Figure 11. Recovery of OMP and TH expression in the OB. AC, Immunohistochemistry for OMP in ipsilateral OB from LPS:10w $(\boldsymbol{A})$, LPS:10w+NT:10w $(\boldsymbol{B})$, and LPS:10w+NC:10w (C). OMP expression recovered in LPS:10w+NT:10w $(\boldsymbol{B})$ and LPS:10w+NC:10w $(\boldsymbol{C})$ compared with that in the LPS:10w $(\boldsymbol{A})$. $\boldsymbol{D}-\boldsymbol{F}$, Immunohistochemistry for TH expressed by some juxtaglomerular cells. TH expression recovered in LPS:10w+NT:10w $(\boldsymbol{E})$ but further decreased in LPS:10w+NC:10w $(\boldsymbol{F})$ compared with that in the LPS:10w $(\boldsymbol{D})$. Nuclei were stained with nuclear fast red. Scale bars: $200 \mu \mathrm{m}$.

differentially affected, both LPS and NC treatments induced shrinkage of the EPL, particularly the sEPL. The superficial one-third of the EPL receives projections from tufted cells, whereas the deeper layers comprise dendrodendritic synapses from mitral cells. Thus, neuronal circuits involving tufted cells are preferentially affected by both chronic nasal inflammation and long-term odor deprivation.

The mechanism for sEPL shrinkage likely involved a loss of OSN activity. OSN activity is eliminated by odor deprivation via NC, while it may not be completely eliminated as a result of inflammation in LPS-treated mice. This idea is supported by the results showing that NC had a much greater effect on the SEPL area than LPS treatment, and induced further shrinkage of $M C L+I P L$ and GCL. NC also reduced TH expression in the GL to a greater extent than LPS treatment, indicating that the odor-induced activity of juxtaglomerular cells was much lower in the NC mice than in the LPS-treated mice.

The results from a previous study suggested that glial activation and proinflammatory cytokines, a hallmark of neuroinflammation, contribute to EPL shrinkage (HasegawaIshii et al., 2019). However, the findings from the present study failed to support this and further demonstrated that this does not occur with odor deprivation. Moreover, the EPL did not recover but rather underwent further shrinkage in the absence of odor input despite the alleviation of inflammation after the cessation of LPS administration. These data suggest that a reduction in glial activation does not contribute to recovery in the OB. By contrast, activated glial cells may support tissue homeostasis in the OB. In the present study, IL-10, an anti-inflammatory cytokine, was upregulated in LPS:10w and NC:10w. Given that the tufted cells were vulnerable to nasal inflammation and odor deprivation, IL-10 may be produced by some cells to protect against the degeneration of tufted cells. Several previous reports indicated that astrocytes have the ability to release IL-10 to protect brain tissue when they are stimulated
(Mizuno et al., 1994; Sawada et al., 1995; Rasley et al., 2006). The upregulated GFAP mRNA expression shows that astrocytes react to nasal inflammation and also odor deprivation. The reactive astrocytes may release IL-10 to maintain OB homeostasis. Within a damaged nasal cavity, for example, Staphylococcus aureus can infiltrate the ONL and GL but not deeper inner layers, possibly because of the actions of activated microglia at the periphery of the $\mathrm{OB}$ (Herbert et al., 2012). Activated microglia produce osteopontin, a neuroprotective cytokine, which acts on CD44 expressed by hippocampal neurons and astrocytes following kainic acid-induced excitotoxic hippocampal injury. This signaling is essential for the neuroprotection and remodeling of hippocampal tissue (Hasegawa-lshii et al., 2011). Thus, the activated microglia in mice with chronic nasal inflammation in the present study may similarly protect the inner layers of the $\mathrm{OB}$ (i.e., the $\mathrm{MCL}, \mathrm{IPL}$, and $\mathrm{GCL}$ ) from atrophy.

Although the GL and EPL completely recovered from atrophy only in the presence of odor input, the abatement of nasal inflammation resulted in only partial recovery of OSNs, which occurred regardless of odor input. This suggests that odor stimulation is not necessary for OSNs to regenerate, mature, and project axons, but is required for $\mathrm{OB}$ neurons to form and maintain synapses. An intriguing result is that the recovery of OSNs was more efficient in LPS: $10 w+N C: 10 w$ than in LPS:10w+NT:10w. When we examined the expression of OMP, calretinin, and GAP43 in the OE, the number of GAP43-positive and calretinin-positive OSNs increased in NC:3w (Extended Data Fig. 8-1A,B), and the increase in the number of calretinin-positive OSNs was more prominent on the closed side in NC:10w than on the open side (Extended Data Fig. 8-1C,D). In the OE, calretinin is expressed by intermediate cells during OSN development (Wei et al., 2013), and we observed a significant increase in the number of calretinin-positive OSNs on the ipsilateral side in LPS:10w+ NC:10w compared with that on the contralateral side and to the ipsilateral side of the OBs in LPS:10w+NT:10w (Extended Data Fig. 8-1E-G). This result suggests that NC stimulates the proliferation of basal cells and promotes the turnover of the OSNs, representing a mechanism underlying the better recovery of the OE in LPS:10w+NC: $10 w$ than in LPS:10w+NT:10w.

OB atrophy induced by odor deprivation also occurs in neonates. NC in neonatal mice significantly reduces the number of granule and external tufted cells but not mitral cells, which develop earlier (Brunjes, 1985, 1994; Frazier and Brunjes, 1988). Thus, there may be a critical neonatal period during which OSN activity supports the survival of granule and external tufted cells and their integration into the neuronal circuit. Another study in which the nostrils of rats were closed on postnatal day 1 and reopened on postnatal day 20 showed that the OBs recovered from atrophy and that the area of the layer returned to normal (Cummings et al., 1997). However, the external tufted cells were not quantified in that study. Further studies examining the SEPL ratio after naris reopening will help to elucidate the mechanisms underlying OSN activity-dependent $\mathrm{OB}$ atrophy and recovery.

Our results suggest that in adults, the dendrites of tufted cells retain plasticity according to OSN activity. In 

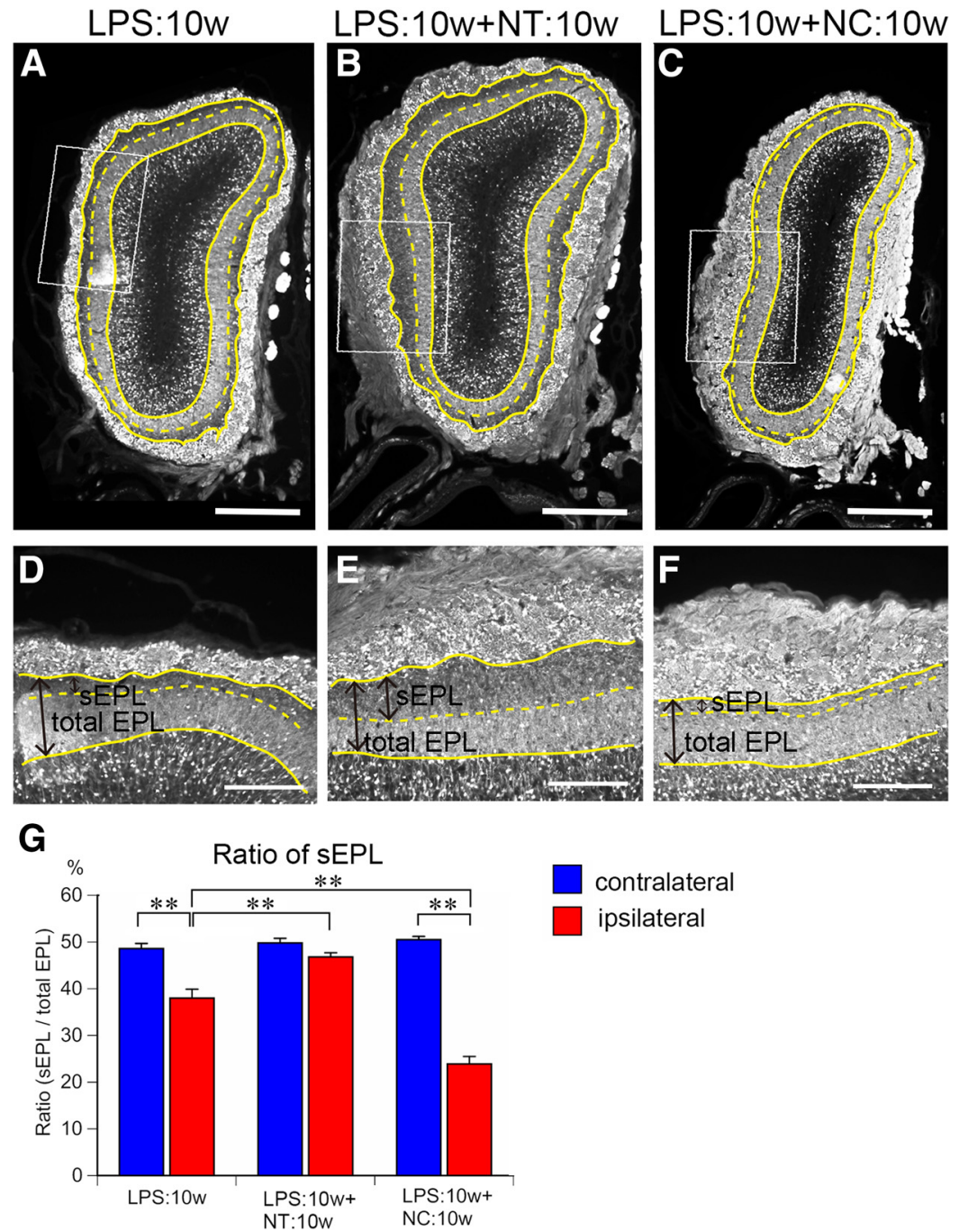

$\square$ contralateral ipsilateral

Figure 12. Recovery of sEPL in the presence or absence of odor input. $\boldsymbol{A}-\boldsymbol{F}$, Coronal sections of ipsilateral OBs stained for calretinin. Solid yellow lines represent the borders of GL and EPL or EPL and MCL. Dotted yellow lines represent the borders of sEPL and dEPL. $\boldsymbol{D}-\boldsymbol{F}$, Magnified views of EPL indicated by rectangles in $\boldsymbol{A}-\boldsymbol{C}$. The sEPL area shrank in LPS:10w $(\boldsymbol{A})$ and recovered in the presence $(\boldsymbol{B})$ but not in the absence $(\boldsymbol{C})$ of odor input. Scale bars: $500 \mu \mathrm{m}(\boldsymbol{A}-\boldsymbol{C})$ and $200 \mu \mathrm{m}(\boldsymbol{D}-\boldsymbol{F})$. G, Graph presents the ratios of sEPL to total EPL. The ratios of sEPL to total EPL significantly decreased in ipsilateral EPL in LPS:10w and LPS:10w+NC:10w, but not in the LPS:10w $+\mathrm{NT}: 10 \mathrm{w} ; * * p<0.01$.

developing neurons, neuronal activity facilitates dendritic stabilization, and the BDNF signaling through TrkB appears to be an important mediator of this effect (Imamura and Greer, 2009). Transgenic mice with lacZ expressed under the control of the BDNF promoter revealed that BDNF is expressed by a subset of interneurons and external tufted cells, but not by mitral cells, in the adult OB (Clevenger et al., 2008). Since TrkB is expressed by both mitral and tufted cells, the differential expression of the BDNF may be a factor underlying their differing dendrite plasticity. In the adult brain, tufted cells are more sensitive to odor inputs and fire in rhythm with respiration (Nagayama et al., 2004; Fukunaga et al., 2012; Igarashi et al., 2012), suggesting that tufted cell circuit is poised to monitor the chemicals and odors in the environment. The high plasticity of tufted cells may be indispensable to monitor the outer chemical environment.

The impact of odor deprivation may not be limited to the $\mathrm{OB}$, because the anterior olfactory nucleus also shrinks
(Barbado et al., 2001) and semilunar cells of the piriform cortex undergo apoptosis in response to odor deprivation (López-Mascaraque and Price, 1997; Leung and Wilson, 2003). Further studies are needed to address whether cortical areas and neurons highly vulnerable to the effects of nasal inflammation and/or odor deprivation predominantly receive input from tufted cells, and whether they are associated with the circuitry changes observed in the OB. A greater understanding of the extent to which nasal inflammation and odor deprivation impact neurons will clarify the relationship between the olfactory system and whole brain functions as well as the association between nasal inflammation and neurologic disorders.

\section{References}

Ahmad N, Zacharek MA (2008) Allergic rhinitis and rhinosinusitis. Otolaryngol Clin North Am 41:267-281.

Barbado MV, Briñón JG, Weruaga E, Porteros A, Arévalo R, Aijón J, Alonso JR (2001) Volumetric changes in the anterior olfactory 
nucleus of the rat after neonatal olfactory deprivation. Exp Neurol 171:379-390.

Bedolla-Barajas M, Morales-Romero J, Pulido-Guillén NA, RoblesFigueroa M, Plascencia-Domínguez BR (2017) Rhinitis as an associated factor for anxiety and depression amongst adults. Braz J Otorhinolaryngol 83:432-438.

Brunjes PC (1985) Unilateral odor deprivation: time course of changes in laminar volume. Brain Res Bull 14:233-237.

Brunjes PC (1994) Unilateral naris closure and olfactory system development. Brain Res Brain Res Rev 19:146-160.

Cho JY, Min N, Franzen L, Baker H (1996) Rapid down-regulation of tyrosine hydroxylase expression in the olfactory bulb of naris-occluded adult rats. J Comp Neurol 369:264-276.

Chou KL, Koeppe RA, Bohnen NI (2011) Rhinorrhea: a common nondopaminergic feature of Parkinson's disease. Mov Disord 26:320-323.

Clevenger AC, Salcedo E, Jones KR, Restrepo D (2008) BDNF promoter-mediated beta-galactosidase expression in the olfactory epithelium and bulb. Chem Senses 33:531-539.

Cummings DM, Henning HE, Brunjes PC (1997) Olfactory bulb recovery after early sensory deprivation. J Neurosci 17:7433-7440.

Dando SJ, Mackay-Sim A, Norton R, Currie BJ, St John JA, Ekberg JA, Batzloff M, Ulett GC, Beacham IR (2014) Pathogens penetrating the central nervous system: infection pathways and the cellular and molecular mechanisms of invasion. Clin Microbiol Rev 27:691-726.

Daulatzai MA (2015) Olfactory dysfunction: its early temporal relationship and neural correlates in the pathogenesis of Alzheimer's disease. J Neural Transm (Vienna) 122:1475-1497.

Doty RL (2008) The olfactory vector hypothesis of neurodegenerative disease: is it viable? Ann Neurol 63:7-15.

Doty RL (2012) Olfaction in Parkinson's disease and related disorders. Neurobiol Dis 46:527-552.

Frazier LL, Brunjes PC (1988) Unilateral odor deprivation: early postnatal changes in olfactory bulb cell density and number. J Comp Neurol 269:355-370.

Fukunaga I, Berning M, Kollo M, Schmaltz A, Schaefer AT (2012) Two distinct channels of olfactory bulb output. Neuron 75:320-329.

Hasegawa-Ishii S, Takei S, Inaba M, Umegaki H, Chiba Y, Furukawa A, Kawamura N, Hosokawa M, Shimada A (2011) Defects in cytokine-mediated neuroprotective glial responses to excitotoxic hippocampal injury in senescence-accelerated mouse. Brain Behav Immun 25:83-100.

Hasegawa-Ishii S, Shimada A, Imamura F (2017) Lipopolysaccharide-initiated persistent rhinitis causes gliosis and synaptic loss in the olfactory bulb. Sci Rep 7:11605.

Hasegawa-Ishii S, Shimada A, Imamura F (2019) Neuroplastic changes in the olfactory bulb associated with nasal inflammation in mice. J Allergy Clin Immunol 143:978-989.e3.

Henegar JR, Maruniak JA (1991) Quantification of the effects of longterm unilateral naris closure on the olfactory bulbs of adult mice. Brain Res 568:230-234.

Herbert RP, Harris J, Chong KP, Chapman J, West AK, Chuah MI (2012) Cytokines and olfactory bulb microglia in response to bacterial challenge in the compromised primary olfactory pathway. $\mathrm{J}$ Neuroinflammation 9:109.

Igarashi KM, leki N, An M, Yamaguchi Y, Nagayama S, Kobayakawa K, Kobayakawa R, Tanifuji M, Sakano H, Chen WR, Mori K (2012) Parallel mitral and tufted cell pathways route distinct odor information to different targets in the olfactory cortex. J Neurosci 32:7970-7985.

Imamura F, Greer CA (2009) Dendritic branching of olfactory bulb mitral and tufted cells: regulation by TrkB. PLoS One 4:e6729.

Imamura F, Hasegawa-Ishii S (2016) Environmental toxicants-induced immune responses in the olfactory mucosa. Front Immunol 7:475.

Kim DH, Han K, Kim SW (2016) Relationship between allergic rhinitis and mental health in the general Korean adult population. Allergy Asthma Immunol Res 8:49-54.
Leung CH, Wilson DA (2003) Trans-neuronal regulation of cortical apoptosis in the adult rat olfactory system. Brain Res 984:182-188.

Li J, Gu CZ, Su JB, Zhu LH, Zhou Y, Huang HY, Liu CF (2016) Changes in olfactory bulb volume in Parkinson's disease: a systematic review and meta-analysis. PLoS One 11:e0149286.

López-Mascaraque L, Price JL (1997) Protein synthesis inhibitors delay transneuronal death in the piriform cortex of young adult rats. Neuroscience 79:463-475.

Malz CR, Knabe W, Kuhn HJ (2000) Pattern of calretinin immunoreactivity in the main olfactory system and the vomeronasal system of the tree shrew, Tupaia belangeri. J Comp Neurol 420:428-436.

Maruniak JA, Lin PJ, Henegar JR (1989a) Effects of unilateral naris closure on the olfactory epithelia of adult mice. Brain Res 490:212-218.

Maruniak JA, Taylor JA, Henegar JR, Williams MB (1989b) Unilateral naris closure in adult mice: atrophy of the deprived-side olfactory bulbs. Brain Res Dev Brain Res 47:27-33.

Maruniak JA, Henegar JR, Sweeney TP (1990) Effects of long-term unilateral naris closure on the olfactory epithelia of adult mice. Brain Res 526:65-72.

Mizuno T, Sawada M, Marunouchi T, Suzumura A (1994) Production of interleukin- 10 by mouse glial cells in culture. Biochem Biophys Res Commun 205:1907-1915.

Nagayama S, Takahashi YK, Yoshihara Y, Mori K (2004) Mitral and tufted cells differ in the decoding manner of odor maps in the rat olfactory bulb. J Neurophysiol 91:2532-2540.

Negoias S, Croy I, Gerber J, Puschmann S, Petrowski K, Joraschky P, Hummel T (2010) Reduced olfactory bulb volume and olfactory sensitivity in patients with acute major depression. Neuroscience 169:415-421.

Rasley A, Tranguch SL, Rati DM, Marriott I (2006) Murine glia express the immunosuppressive cytokine, interleukin-10, following exposure to Borrelia burgdorferi or Neisseria meningitidis. Glia 53:583-592.

Rochet M, El-Hage W, Richa S, Kazour F, Atanasova B (2018) Depression, olfaction, and quality of life: a mutual relationship. Brain Sci 8:80.

Rombaux P, Potier H, Bertrand B, Duprez T, Hummel T (2008) Olfactory bulb volume in patients with sinonasal disease. Am J Rhinol 22:598-601.

Rottstädt F, Han P, Weidner K, Schellong J, Wolff-Stephan S, Strauß T, Kitzler H, Hummel T, Croy I (2018) Reduced olfactory bulb volume in depression-a structural moderator analysis. Hum Brain Mapp 39:2573-2582.

Sawada M, Suzumura A, Marunouchi T (1995) Cytokine network in the central nervous system and its roles in growth and differentiation of glial and neuronal cells. Int J Dev Neurosci 13:253-264.

Schlosser RJ, Storck K, Cortese BM, Uhde TW, Rudmik L, Soler ZM (2016) Depression in chronic rhinosinusitis: a controlled cohort study. Am J Rhinol Allergy 30:128-133.

Siopi E, Denizet M, Gabellec MM, de Chaumont F, Olivo-Marin JC, Guilloux JP, Lledo PM, Lazarini F (2016) Anxiety- and depressionlike states lead to pronounced olfactory deficits and impaired adult neurogenesis in mice. J Neurosci 36:518-531.

Wei H, Lang MF, Jiang X (2013) Calretinin is expressed in the intermediate cells during olfactory receptor neuron development. Neurosci Lett 542:42-46.

Woo CC, Hingco EE, Taylor GE, Leon M (2006) Exposure to a broad range of odorants decreases cell mortality in the olfactory bulb. Neuroreport 17:817-821.

Yamaguchi M, Mori K (2005) Critical period for sensory experiencedependent survival of newly generated granule cells in the adult mouse olfactory bulb. Proc Natl Acad Sci USA 102:9697-9702.

Yuan TF, Slotnick BM (2014) Roles of olfactory system dysfunction in depression. Prog Neuropsychopharmacol Biol Psychiatry 54:26-30. 